\title{
Human fetal bone cells in delivery systems for bone engineering
}

\author{
Diene M. H. Tenorio ${ }^{1,2,3}$, Corinne Scaletta ${ }^{1,2}$, Sandra Jaccoud ${ }^{1,2}$, Nathalie Hirt-Burri ${ }^{1}$, \\ Dominique P. Pioletti ${ }^{2}$, Bertrand Jaques ${ }^{4}$ and Lee Ann Applegate ${ }^{1,2 *}$ \\ ${ }^{1}$ Cellular Therapy Unit, Department of Musculoskeletal Medicine, University Hospital of Lausanne, Switzerland \\ ${ }^{2}$ Laboratory of Biomechanical Orthopaedics, Ecole Polytechnique Fédérale de Lausanne, Switzerland \\ ${ }^{3}$ Department of Histology, Federal University of Alagoas, Brazil \\ ${ }^{4}$ Department of Maxillofacial Surgery, University Hospital of Lausanne, Switzerland
}

\begin{abstract}
The aim of this study was to culture human fetal bone cells (dedicated cell banks of fetal bone derived from 14 week gestation femurs) within both hyaluronic acid gel and collagen foam, to compare the biocompatibility of both matrices as potential delivery systems for bone engineering and particularly for oral application. Fetal bone cell banks were prepared from one organ donation and cells were cultured for up to 4 weeks within hyaluronic acid (Mesolis ${ }^{\circledR}$ ) and collagen foams (TissueFleece ${ }^{\circledR}$ ). Cell survival and differentiation were assessed by cell proliferation assays and histology of frozen sections stained with Giemsa, von Kossa and ALP at 1, 2 and 4 weeks of culture. Within both materials, fetal bone cells could proliferate in three-dimensional structure at $\sim 70 \%$ capacity compared to monolayer culture. In addition, these cells were positive for ALP and von Kossa staining, indicating cellular differentiation and matrix production. Collagen foam provides a better structure for fetal bone cell delivery if cavity filling is necessary and hydrogels would permit an injectable technique for difficult to treat areas. In all, there was high biocompatibility, cellular differentiation and matrix deposition seen in both matrices by fetal bone cells, allowing for easy cell delivery for bone stimulation in vivo. Copyright $\odot 2011$ John Wiley \& Sons, Ltd.
\end{abstract}

Received 6 January 2010; Accepted 22 September 2010

Keywords bone regeneration; cell therapy; wound healing; cell activation; hydrogels; hyaluronic acid; scaffolds

\section{Introduction}

Bone loss is still a clinical problem that attracts the interest of many investigations in order to determine an alternative for bone replacement. Specifically for oral application, bone tissue is necessary to supply areas to support dental implants or in cases of periodontal disease, which requires regeneration of functional bone tissue (Patino et al., 2002; Yildirim et al., 2000; Mendes et al, 2008). Classical surgery techniques, such

\footnotetext{
*Correspondence to: Lee Ann Applegate, Cellular Therapy Unit, Department of Musculoskeletal Medicine, Service of Plastic and Reconstructive Surgery, University Hospital of Lausanne, CHUV PAV 03, CH-1011 Lausanne, Switzerland.

E-mail:Lee.Laurent-Applegate@chuv.ch
}

as autograft, allograft and xenograft for bone tissue repair, consist of invasive traumatic procedures with a risk of contamination and immunological response complications (Younger and Chapman, 1989; Strong et al., 1996).

To overcome this problem, cell therapy has been proposed as a less invasive alternative for bone engineering. Several cell types have been investigated to be used in cell therapy: embryonic stem cells (ESCs), umbilical cord cells, fetal cells and adult stem cells from bone marrow haematopoietic stem cells (HSCs) and mesenchymal stem cells (MSCs), along with adipose tissue, platelets, placenta and amniotic fluid cells (Guillot et al., 2007; Bianco and Robey, 2001; Bullard et al., 2003; Kaviani et al., 2001; Kaviani et al., 2002; Tobita et al., 2008; Kruyt et al., 2008). As for any application in tissue engineering, the cell origin and type are 
essential aspects in bone tissue engineering (Guillot et al., 2007; Pioletti et al., 2006; Quintin et al., 2007). Stem cells and cell populations derived at different stages of development are associated with terminology that can be quite confusing. Despite the diversity of cell source, each type of cell requires different methods to manipulate its differentiation and self-renewal capabilities for specific therapies, with various advantages and disadvantages (Table 1, Figure 1).

The relatively simple manipulation of fetal cells in relation to their collection, culture expansion and storage has made fetal cells an attractive choice for cell therapy, as they are tissue-specific, thereby eliminating rigorous population selection and differentiation by multiple growth factors. In addition, fetal cells have the advantage of high proliferation and sustaining a differentiation state and potential for mineralization activity in vitro (Montjovent et al., 2004). Unlike ESCs, fetal cells do not form tumours and seem to lack immunogenicity when transplanted (Montjovent et al., 2009). Due to the rapid cellular growth and minimum nutrient requirements of fetal bone cells, fetal cell banking can be established to assure high levels of consistency. In contrast to MSCs, fetal bone cells do not require feeder layers for growth or specific growth factors for differentiation. One organ donation is capable of producing a master cell bank (MCB) that would be available for hundreds of thousands of patient treatments. The fully defined consistent cell bank could easily be assessed for safety concerning any potential virus and pathogens in parallel to the original organ donation where serology and pathology are accomplished (Quintin et al., 2007). Among the major challenges that will assure many patients benefiting from bone tissue engineering in the future is not only related to the choice of cell type, their isolation and proliferation but equally to a biocompatible delivery system for the chosen cell type.

Previous results from Montjovent et al. (2005) demonstrate that a composite made of poly-L-lactic acid and $\beta$-tricalcium phosphate particles used as scaffolds to seed fetal bone cells can offer suitable conditions for osteoblasts to achieve full differentiation, due to the high osteogenic potential of these cells. Furthermore, in vivo studies using dedicated fetal bone cell banks with solid matrix have shown significant promotion of bone growth using two different model systems (Montjovent et al., 2005, 2007, 2008).

Several hydrogels, whether synthetic or naturally derived, have extensive use in medicine, pharmaceutical and basic sciences (Drury and Mooney, 2003). Hydrogels provide a hydrated space and a mechanical carrier for cell transplantation and tissue engineering. The interest for many studies on hydrogels and collagen-based matrices is the use of these materials for clinical injectable cell delivery directly within defect areas for tissue engineering to minimize surgical procedures (Patino et al., 2002; Drury and Mooney, 2003; Lutolf and Hubbell, 2005; Alsberg et al., 2001; Weinand et al., 2006). Collagen has been incorporated into a variety of medical devices and has been used for multiple proposes (Mast et al.,1993). For dental applications, resorbable forms of collagen have been used to dress oral wounds, for closure of graft and extraction sites, for delivery of autologous bone residues and to promote healing (Wang, 1998). In all, biocompatible biomaterials need to be available in order to provide an extracellular matrix environment for bone cell differentiation and release. In this study, we evaluated the use of a hydrogel of hyaluronic acid (HA; Mesolis ${ }^{\circledR}, \mathrm{CE}$ marked) and a collagen foam (TissueFleece ${ }^{\circledR}$, CE marked medical device) as human fetal bone cell delivery systems to verify their biological behaviour and biocompatibility for bone engineering.

\section{Materials and methods}

\subsection{Bone donations and cell bank synthesis}

Cell banks were established in the University Hospital of Lausanne from a fetal femur bone biopsy at 14 weeks of gestation (14 wFB), obtained after pregnancy termination with informed and written consent and approval from the local Medical School Ethics Committee (Protocol No. 51/01).

Cell banks were prepared from $0.5 \mathrm{~cm}^{3}$ bone biopsy at passages $2-3$. The detailed procedure has been described previously for fetal skin (de Buys Roessingh et al., 2006; Quintin et al., 2007) and specific conditions for the present study are described briefly. A bone biopsy was dissected into $0.5 \mathrm{~mm}^{3}$ fragments, then approximately five fragments were distributed into $10 \mathrm{~cm}$ culture dishes previously scored deeply with a sterile scalpel in a chequer-board pattern under a laminar flow hood. A small quantity of culture medium containing DMEM with $10 \%$ fetal bovine serum (FBS; Hyclone) was placed around each fragment to avoid floating of the tissues for the first $24 \mathrm{~h}$; rare floating tissues could be overlaid with a sterile coverslip for the first $24 \mathrm{~h}$ if necessary. After the first $24 \mathrm{~h}$, an additional $8 \mathrm{ml}$ culture medium were added onto each $10 \mathrm{~cm}$ plate and this was changed twice weekly before passage. Cell cultures were grown at $37^{\circ} \mathrm{C}$ in a humidified atmosphere of $95 \%$ air $/ 10 \% \mathrm{CO}_{2}$.

When cell growth had advanced after approximately 1-2 weeks for fetal bone cells, the dishes of tissue and cells were trypsinized [0.25\% trypsin: $0.1 \%$ ethylene diaminetetra-acetic acid (EDTA)]. At this point, some fetal bone cells were frozen into individual units in liquid nitrogen and others were passaged at 2000 cells $/ \mathrm{cm}^{2}$ for producing the MCB, as described previously by Quintin et al. (2007).

\subsection{Fetal bone cell growth within hydrogels and collagen foam}

Cell growth was measured at various time points following culture of fetal bone cells at passage 4 within hydrogels and collagen matrix. Cells in hydrogels 


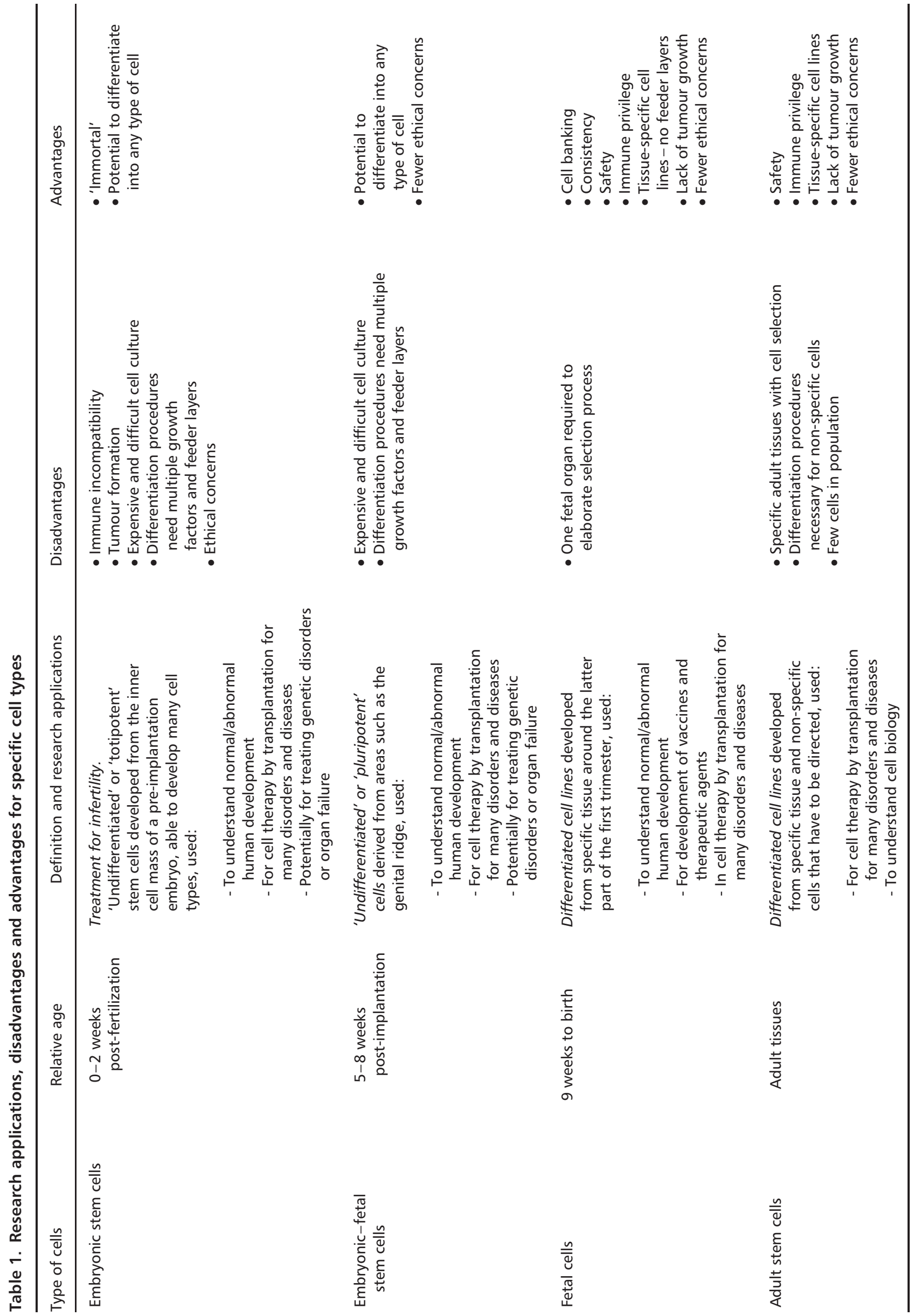




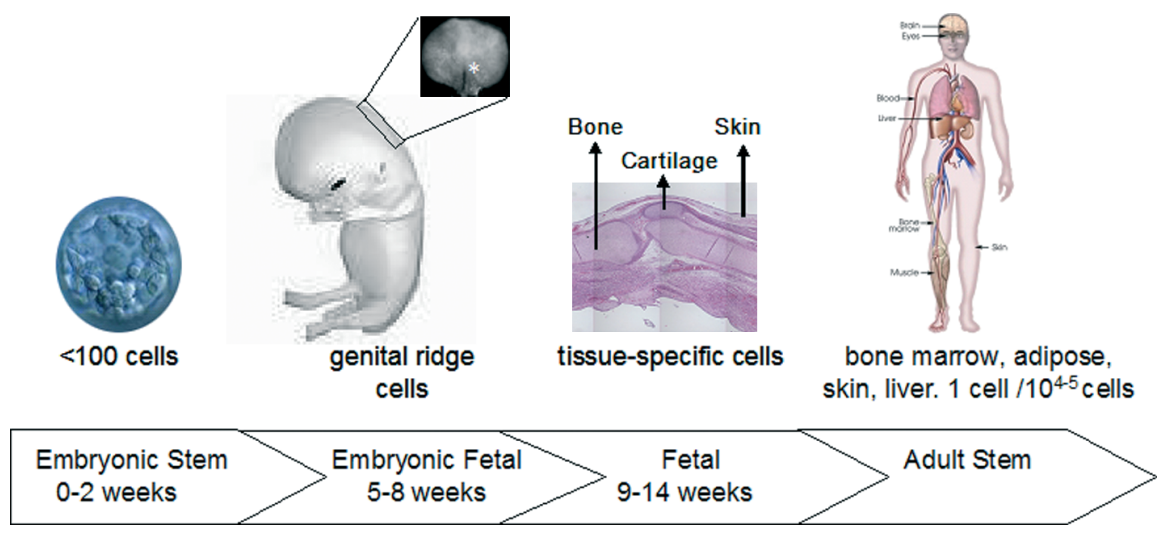

Figure 1. Development stage, cell type and source of cells currently used in cellular therapy. Embryonic stem cells are derived from embryos at an early stage when $<100$ cells are present, followed by fetal stem cells that are taken from the genital ridge section from 5-8 weeks of gestation (drawing taken from: http://www.wikipedia.org). Tissue-specific fetal cells are taken following 9 weeks of gestation usually up to 14-16 weeks from normal tissue. Adult stem cells can be isolated from most tissue sources (image taken from: http://www.national academics.org/stemcells) but are rare, with only 1 in every $10^{4}-10^{5}$ of total cell volume

and collagens were plated in 24-well (hydrogels) and six-well (collagen) tissue culture dishes at a density of 10000 cells $/ \mathrm{cm}^{2}$ for hydrogels and 50000 and 100000 cells $/ \mathrm{cm}^{2}$ (collagen) in triplicate, respectively. Cell growth medium was over-layered onto the cultures. Following 1, 3, 6 or 7 and 9 days, samples were treated with trypsin + EDTA (Gibco) for hydrogels for $5 \mathrm{~min}$ and with trypsin + EDTA + collagenase (collagenase type II, Clostridium histolyticum, Gibco) for $30 \mathrm{~min}$ at $37^{\circ} \mathrm{C}$ to separate cells individually from each sample. Collagenase treatment completely degraded fibrous collagen to solution form, therefore liberating all the cells in each sample. Cell counts were elaborated with the aid of a haemocytometer and an automatic cell counter and repeated in triplicate.

\subsection{Fetal bone biocompatibility with hyaluronic acid hydrogels and collagen foams}

For hyaluronic acid (HA) hydrogels, we used the hyaluronic acid gel Mesolis ${ }^{\circledR}$ (Anteis SA, Geneva, Switzerland). It was necessary to prepare an agar mould to avoid cellular attachment to the tube and allow threedimensional (3D) growth. The mould was prepared by pipetting $1 \mathrm{ml}$ melted agarose (20\% low-melting agar) inside a $1.5 \mathrm{ml}$ sterile conical Eppendorf centrifuge tube, in which a $0.5 \mathrm{ml}$ sterile conical Eppendorf centrifuge tube was inserted into the liquid agar and allowed to solidify before extracting the $0.5 \mathrm{ml}$ tube, leaving a conical inset (Figure 3). HA hydrogel with 10000 cells/ml were inserted into the agar mould and $100 \mu \mathrm{l}$ culture medium were pipetted onto the surface of each tube; thereafter the medium was changed twice weekly. The cells were grown for 1,2 and 4 weeks in a $37{ }^{\circ} \mathrm{C}$ incubator at $95 \%$ relative humidity and $10 \% \mathrm{CO}_{2}$.

For collagen foam sheet preparations we used TissueFleece ${ }^{\circledR}$ equine collagen sheets of $2 \mathrm{~mm}$ thickness dry weight (Baxter, Switzerland). Fetal bone cells at passages 3 or 4 were placed in $10 \mathrm{ml}$ culture medium and seeded onto the collagen sheets at a density of 10000 cells $/ \mathrm{cm}^{2}$. The sheets containing the fetal bone cells were placed into a $37^{\circ} \mathrm{C}$ incubator at $95 \%$ relative humidity and $10 \% \mathrm{CO}_{2}$. An additional $30 \mathrm{ml}$ culture medium was added $1 \mathrm{~h}$ later. For collagen foams with integrated fetal bone cells, the medium was changed twice weekly with nutrient medium. Fetal bone cell-seeded collagen foams remained at a $2 \mathrm{~mm}$ thickness, like the original dry collagen sheets.

Biocompatibility was also measured by a contact assay, in which cells were cultured within a tissue culture plate where hydrogel or collagen were initially seeded. Cell growth and migration were analysed visually with respect to the hydrogel-collagen foam interface. After 1, 2 and 4 weeks, the samples were stained with Giemsa and photographed (Sony CyberShot DSC-S70, Zeiss macro lens, zoom $\times 6,3.3$ megapixels).

As the hydrogels were transparent, cells that had migrated to the culture plate could easily be photographed to visually assure biocompatibility with the gel over the growth period and to assess efficient cell growth with no change in morphology (Leica DMIL inverse, phasecontrast with ocular $\times 10 / 18$ and objective $\times 10 / 0.22 \mathrm{C}$ Plan PH1).

\subsection{Fetal bone function and matrix deposition}

After 2 and 4 weeks of culture, cells cultivated within the HA hydrogel inside the agar mould and collagen were frozen in liquid nitrogen. Sections $(20 \mu \mathrm{m})$ were cut and the staining procedures were followed after fixation of samples with $4 \%$ formaldehyde. For detection of alkaline phosphatase (ALP) activity, we followed the procedure from Sigma-Aldrich, using p-nitrophenol tablets as substrate (85L3R-1KT). Staining for von Kossa (von Kossa-Silver Nitrate; Sigma-S-6506) was applied to detect clusters of mineralization in the matrix (Bonewald et al., 2003). Staining with Giemsa was performed to observe HA hydrogel characteristics as cell support. For 




Figure 2. Matrix deposition. Histological sections $(20 \mu \mathrm{m})$ of fetal bone cells grown for 2 weeks in HA Mesolis hydrogels or Baxter TissueFleece collagen foams were stained with Giemsa, ALP and von Kossa ( $\times 100)$

von Kossa staining, cells grown in hydrogels and collagen foams were washed twice with PBS following fixation, after which the fixative was removed and the samples put into $5 \%$ silver nitrate solution and then exposed to UV light for $15 \mathrm{~min}$, followed by a deionized water wash. Sodium thiosulphate (5\% in deionized water) was added for $3 \mathrm{~min}$, the samples were washed with deionized water and counterstained with Nuclear fast red (Sigma-Aldrich: red for nuclei) for $3 \mathrm{~min}$. All samples were mounted with coverslips and then photographed (Leica, Leitz DMRB, ocular L Plan $\times 10 / 0.25$ with objectives $\times 10 / 0.25$ N PLAN or $\times 20 / 0.40$ N PLAN).

\section{Results and discussion}

In this study we were interested in evaluating two commercially available materials for human fetal bone cell delivery regarding biocompatibility and bone cell differentiation. In particular, we wanted to test a solid and a liquid-gel scaffold as different clinical situations may need one or the other.

After 2 weeks of culture, fetal bone cells were shown to survive and produce matrix within the two different cell delivery systems, HA hydrogels and collagen foams, providing a 3D structure (Figure 2). Giemsa staining showed the overall ultrastructure of cells integrated throughout the HA hydrogel and collagen foam. Hydrogels permitted the cells to proliferate in a 3D semi-liquid form where the cells remained round in morphology. This was technically possible since the agar moulds were used to ensure that cellular growth only occurred in a 'suspension' within the hydrogels. If the hydrogel was placed in plastic tubes or plates, cell migration towards these surfaces was observed, as these cells prefer, and grow more rapidly in, a monolayer than in suspension, as they have adhesion possibilities.
Cell morphology within the collagen foam permitted a structure similar to that seen when cells are grown in monolayer tissue culture flasks, showing a fibroblastic nature. Rapid adhesion with materials allows a more rapid cellular growth and the development of colonies of cells instead of individual cell growth throughout a matrix. Within the two cell delivery systems, histology showed that these cells had markers for differentiated bone (ALP) and that matrix deposition was evident already (von Kossa). HA hydrogels or collagen foams alone did not take up any residual staining for each technique for histological staining as a control, indicating that it is the cells themselves producing the matrix deposition over time (Figure 2). After 4 weeks of culture, cell growth was still observed and higher matrix deposition was indicated by von Kossa staining around cells and especially throughout the collagen foam further away from individual cells. This time point was mainly to evaluate any potential degrading by-product following long-term cell-matrix association, since matrix deposition was seen already at 2 weeks of 3D culture of fetal bone cells, as shown in Figure 2.

\subsection{Biocompatibility of fetal bone cells with HA hydrogel and collagen foam}

Biocompatibility of fetal bone cells was assessed by evaluating whether human fetal bone cells can survive and proliferate within the HA hydrogel and collagen (Figure 3). Cells grown within hydrogels and also in contact with hydrogels within 24-well plates could be quantified. Visually, the cells were able to proliferate very well, with the same morphology as those cells that were not in contact with hydrogel. The cells were counted at various time points and proliferation was shown to be $70 \%$ of that of cells grown without the presence of hydrogel, which represents the most rapid condition as they are fully in monolayer growth (Figure 4A). Cell growth was seen to increase by three- and five-fold over a 9 day period 


\section{Biocompatibility}

HA hydrogels (Mesolis ${ }^{\circledR}$ )
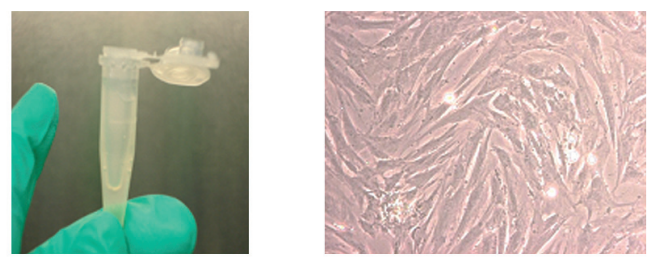

Baxter TissueFleece ${ }^{\circledR}$ 3D sponge

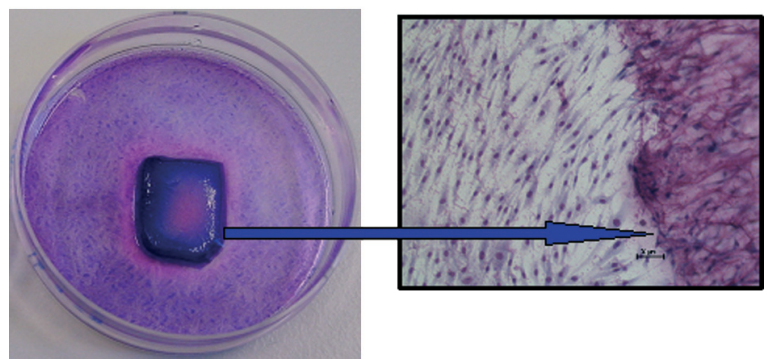

Figure 3. Biocompatibility of fetal bone cells with Mesolis hydrogel and Baxter TissueFleece collagen foams at 4 weeks. (Top) For the hydrogel, it was necessary to use an agar mould (photo of tube preparation) to allow cell growth in 3D structure in suspension and not attaching to plastic surfaces. As the hydrogels were transparent, cells that had migrated to the culture plate could easily be photographed to visually assure biocompatibility with the gel over the growth period and to assess efficient cell growth with no change in morphology. (Bottom) Biocompatibility of fetal bone cells with collagen foam was evaluated by a contact assay in which foams seeded with cells were cultured within a tissue culture plate, cell growth and migration were analysed macroscopically and microscopically and samples were stained with Giemsa and photographed (Sony CyberShot DSC-S70, Zeiss macro lens, zoom $\times 6,3.3$ megapixels) $(\times 100)$. No degradation by-products was observed after 4 weeks

for cells in hydrogel and those grown in monolayer, respectively. Biocompatibility of fetal bone cells was evidenced visually over time without toxicity being observed. Cell morphology remained similar throughout the experiments, with cells having a fibroblastic nature. When fetal bone cells were placed in hydrogels inside plastic tubes or plates that were not tissue-grade quality, the hydrogel provided a coating for these materials to allow rapid cellular attachment. Migration of cells was within $1 \mathrm{~h}$, when all cells were already attached to the hard surfaces. This could be an important trait for cell delivery if the hydrogel were used to simplify delivery of fetal bone cells locally by injection and would assure rapid migration to attach to host bone structures. The migratory and adhesion properties of fetal cells have been shown to be more highly regulated than other cell types used in tissue and cellular therapy to date (Hirt-Burri et al., 2008; Ramelet et al., 2009). Biocompatibility was tested using the agar mould coating to assure no cellular attachment. Although fetal bone cells prefer adhesion for best growth capacity, these cells were seen to grow gradually and remain alive for up to 4 weeks in 'suspension' within the hydrogel, which emphasizes that the hydrogel does
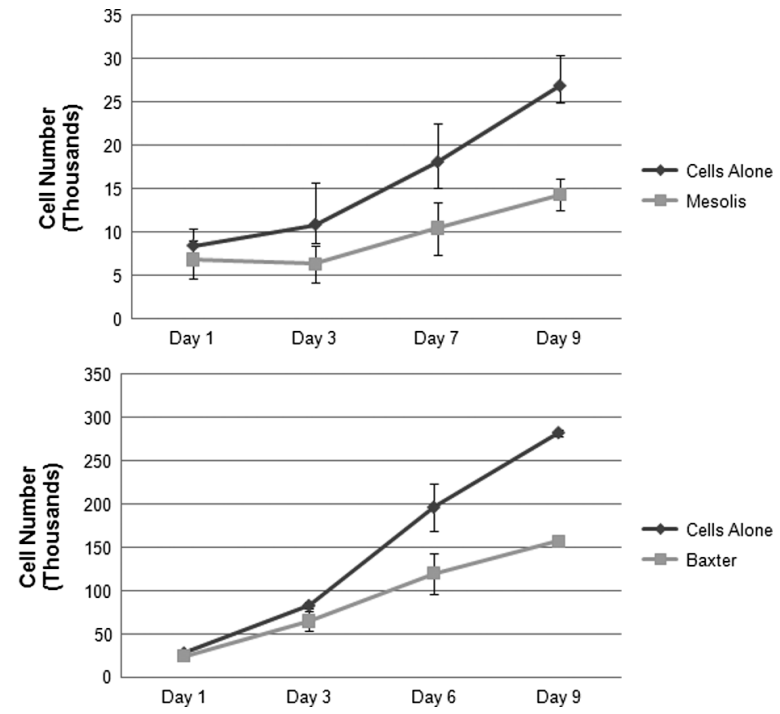

Figure 4. Cell growth. Cell growth is represented in normal cell culture compared to growth in Mesolis hydrogel (top) or Baxter collagen foam (bottom), with cell number as a function of days in culture. Data are representative of three individual experiments in triplicate with associated standard deviation of the mean

not produce degradative by-products affecting cellular growth, even after long periods of contact. Overall, neither hydrogel nor collagen showed degradation or shrinkage over the 4 week period of cellular growth within and did not elicit toxicity to the fetal bone cells. Collagen foam can also provide a support for the cells during the 2 and 4 weeks of cell culture, with no apparent degradation in vitro. Biocompatibility was also seen to be very high, as represented by the contact assay, in which cells are layered around the collagen foam in culture and cells migrated towards the collagen foam and integrated within easily (interface of contact between cells and collagen, Figure 3). Structurally, as it is a solid, collagen foam is easier to manage for histological procedures than HA hydrogel, but both can support the cells and provide a matrix for cell survival, proliferation and differentiation.

The motivation for using commercially available scaffolds was based on the fact that the specific chosen materials are already used in different clinical applications. The translation of the results obtained here to a clinical application would then be easier than with a newly developed scaffold that had not been validated. This aspect is very important when evaluating a combination product (association of a medical device that already has approval with clinical-grade cells) for clinical use, as the association of the two elements should not produce toxic degradation by-products. Therefore, the biocompatibility of each of these scaffolds, showing cell proliferation even after 4 weeks, provides essential information for how live cells interact with their support for delivery.

As the use of fetal cell therapy has already successfully been implemented in clinical trials for skin applications (de Buys Roessingh et al., 2006; Ramelet et al., 2009; Hohlfeld et al., 2005; Applegate et al., 2009), we now 
have the possibility to propose different delivery systems using human fetal bone cells for preclinical assessment.

Overall, the results demonstrated good biocompatility of the two tested scaffolds, namely a hyaluronic acid hydrogel (Mesolis ${ }^{\circledR}$ ) and a collagen foam (TissueFleece ${ }^{\circledR}$ ). Not only were the viability and proliferation of fetal cells observed, but also differentiation toward mature osteoblasts was supported by both scaffolds. These aspects are obviously a prerequisite in the perspective of using a material for cell delivery. The results obtained with the collagen foam are in line with previous studies. Indeed, human fetal bone cells were shown to fully differentiate into mature osteoblasts, as demonstrated by a mineralization assay, when these cells were cultivated in foam made of poly-L-lactic acid and $\beta$-tricalcium phosphate particles (Montjovent et al., 2005). In general, polymer foams seem to be an adequate support for bone formation. The proliferation and differentiation of fetal bone cells cultured in HA hydrogel was indeed more surprising. Osteoblasts or, more generally, fibroblasts are anchorage-dependant for their development. Hydrogel is then a scaffold not offering a strong anchorage to cells, which could be considered to be in a suspension. Not all hydrogels support the differentiation of osteoblasts; for example, fibrin gel has been shown to hamper the proliferation of cells, including MSCs and fetal bone cells.

Surprisingly, the general use of fetal cells in clinics, if properly done, is more straightforward than with MSCs. Indeed, one of the major challenges for assuring that more patients will benefit from cell-based therapies in the future will be the optimization of the choice of cell type, as well as their isolation, proliferation and delivery. The development of MCB from the cell choice provides a major advantage for the creation of a therapeutic biological agent. Fetal cells are easily adapted to this type of development, since only one organ donation is necessary to develop a MCB from a specific tissue such as fetal bone. Fetal cells, unlike many stem cell types, do not need feeder layers or external growth factors for cell differentiation processes. Fetal cells are differentiated cells, since they are derived from specific tissues and organs. Even though stem cells have an advantage in changing and differentiating into multiple cell lineages, it is difficult to assure their final cell type differentiation when placed in vivo. Once MCB can be produced, working cell banks can be set up to establish individual batches of treatments for high numbers of patients (for skin and bone, this can be up to hundreds of thousands of patients from one cell-banking process and from one organ donation). Further, these cell banks can be completely tested for safety regarding sterility, pathogens, adventitious agents and tumorigenicity.

Despite its important potential, the ethical and related emotional aspects of developing a fetal cell therapy should not be underestimated, and in particular there is considerable misinformation about this subject. Although there is high medical support for developing cell-based therapies to reach as many patients as possible, there exists a governing political controversy. In the 1930s, medical doctors and scientists used tissue from voluntary pregnancy interruptions, not only to aid in the understanding of cell biology but also as an important entity in the development of vaccines, by using defined tissue-derived cell lines. The Nobel Prize for Medicine in 1954 was awarded to American immunologists who developed the polio vaccine based on cultures of human fetal cells. Since this time, many other necessary vaccines (rubella, chicken pox, hepatitis A, etc.) have been developed with the use of fetal cell lines, including two primary human diploid cell lines which were originally prepared in the 1960s - the WI-38 (Wistar Institute 38) in 1964 and the MRC-5 (Medical Research Council 5) in 1966. In Switzerland and most countries, fetal tissue is considered as an organ donation by law (Heinonen et al., 2005; Trommelmans et al., 2007, 2008; Pfeffer and Kent, 2006; Kent and Pfeffer, 2006). This process is highly regulated, including federal approval for tissue biopsy, stocking and transplantation and ethics committee approval of the procedure, with elaborate protocols for informed consent of the donor (Commission of the European Communities Proposal for a Regulation of the European Parliament and of the Council on Advanced Therapy Medicinal Production and Amending Directive 2001/83/EC and Regulation (EC) No. 726/2004; 'Setting standards of quality and safety for the donation, procurement, testing, processing, storage, and distribution of human tissues and cells'. Off $J$ European Union 2001; L 102/48).

Not only is the cell choice pertinent, but equally important will be the delivery system of the cell type and its interaction with these scaffolds to assure biocompatibility and that no toxic degradation by-products are formed. Collagen foams, similar to those used for skin regeneration, are used routinely by dental surgeons. Before closing wounds for dental implants, many dental surgeons place the bone pulp that is removed from the drill-hole procedure onto a collagen sponge before closing the wound. In the same manner, collagen foams provide a first measure of cell presentation. For better stimulation of osseo-intergration, fetal bone cells could be delivered during the closing procedure to reduce the non-load period following the dental implant. Further stimulation of tissue repair could be delivered by an injectable form of cell delivery, which could be in a hydrogel format. We have seen that fetal bone cells adapt well to hyaluronic acid and have a viscosity that is readily injected through very small needle bores without affecting cell survival. Hyaluronic acid (HA) is a molecule abundant in the human body. Its molecular architecture allows the crosslinking to proteoglycan and collagen which makes HA useful as a carrier for cells. Additionally, HA facilitates cell migration to the surrounding tissue that could allow rapid cell delivery for bone repair in vivo (Drury and Mooney, 2003; Weinand et al., 2006). Collagen-based materials have been used as a membrane barrier in oral application. Collagen is a very useful material because of its biocompatibility, absorption, chemotactic qualities and also the capability of enhancing 
migration and filling space (Patino et al., 2002). Therefore, the vast functional diversity of these two cell carriers could potentially be used together for overall treatment.

\section{Conclusions}

In this study, human fetal bone cell delivery was investigated using two different biomaterials. The biocompatibility and cell differentiation by observing cellular matrix deposition was evaluated in 3D cell culture. When using equine collagen foam and hyaluronic acid hydrogel, not only were the viability and proliferation of fetal cells observed but also differentiation toward mature osteoblasts was supported by both scaffolds. Collagen foam, due to its resistant properties, could be used as a cell carrier for initial treatment in areas that need a bone support and a hyaluronic acid gel as a sustained therapy for rapid and localized cell delivery to damaged host bone when additional bone stimulation would be needed. Importantly, we observed that both collagen and HA sustained their structure over the 4 weeks of culture and provided a support for cell survival, proliferation and differentiation. Overall, cellular therapies could provide an interesting alternative to single growth factor delivery (Nikolidakis et al., 2009) for bone regeneration, given in non-physiological dosages to the patient.

\section{Acknowledgements}

The authors wish to thank Baxter (Switzerland) for the donation of the matrix (TissueFleece ${ }^{\circledR}$ ) for these studies, and Anteis SA (Geneva, Switzerland) for donation of HA Mesolis ${ }^{\circledR}$. Particular thanks to Dr. Samuel Gavard and Dr. Cyrille Vinchon from Anties for continued discussions. These studies were supported by Coordination of Perfectioning of Staff of Superior Level (CAPES) by the Ministry of Education and Culture of Brazil, by the Inter-institutional Centre of Translational Biomechanics (EPFL-CHUV-DAL) and in part by the SANTE Foundation.

\section{References}

Alsberg E, Anderson KW, Albeiruti A, et al. 2001; Cell-interactive alginate hydrogels for bone tissue engineering. J Dent Res 80: 2025-2029.

Applegate LA, Scaletta C, Hirt-Burri N, et al. 2009; Whole-cell bioprocessing of human fetal cells for tissue engineering of skin. Skin Pharmacol Physiol 22: 63-73.

Bianco P, Robey PG. 2001; Stem cells in tissue engineering. Nature 414: 118-122.

Bonewald LF, Harris SE, Rosser J, et al. 2003; Von Kossa staining alone is not sufficient to confirm that mineralization in vitro represents bone formation. Calcif Tissue Int 72: 537-547.

Bullard KM, Longaker MT, Lorenz HP. 2003; Fetal wound healing: current biology. World J Surg 27: 54-61.

de Buys Roessingh A, Hohlfeld J, Scaletta C, et al. 2006; Development and characterization of a fetal skin bank for tissue engineering and its clinical application for acute and chronic wounds. Cell Transpl 15: $823-834$.

Drury JL, Mooney DJ. 2003; Hydrogels for tissue enginnering: scaffold design variables and applications. Biomaterials 24: 4337-4351.

Guillot PV, Cui W, Fisk NM, et al. 2007; Stem cell differentiation and expansion for clinical applications of tissue engineering. J Cell Mol Med 11: 935-944.

Heinonen M, Oila O, Nordstrom K. 2005; Current issues in the regulation of human tissue-engineering products in the European Union. Tissue Eng 11: 1905-1911.

Hirt-Burri N, Scaletta C, Gerber S, et al. 2008; Wound-healing gene family expression differences between fetal and foreskin cells used for bioengineered skin substitutes. Artif Organs 32: 509-518.
Hohlfeld J, de Buys Roessingh A, HirtBurri N, et al. 2005; Tissue engineered fetal skin constructs for paediatric burns. Lancet 366: 840-842.

Kaviani A, Perry T, Dzakovic A, et al. 2001; The amniotic fluid as a source of cells for fetal tissue engineering. J Pediatr Surg 36: 1662-1665.

Kaviani A, Perry T, Burnes CM, et al. 2002; The placenta as a cell source in fetal tissue engineering. J Pediatr Surg 37: 995-999.

Kent J, Pfeffer N. 2006; Regulation the collection and use of fetal stem cells. $\mathrm{Br}$ Med $J$ 332: 866-867.

Kruyt M, De Bruijn J, Rouwkema J, et al. 2008; Analysis of the dynamics of bone formation, effect of cell seeding density, and potential of allogeneic cells in cellbased bone tissue engineering in goats. Tissue Eng 6: 1081-1088.

Lutolf MP, Hubbell JA. 2005; Synthetic biomaterials as instructive extracellular microenviroments for morphogenesis in tissue engineering. Nat Biotechnol 23: 47-53.

Mast BA, Diegelmann RF, Krummel TM, et al. 1993; Hyaluronic acid modulates proliferation, collagen and protein synthesis of cultured fetal fibroblasts. Matrix 13: 441-446.

Mendes RM, Silva GAB, Lima MF, et al. 2008; Sodium hyaluronate accelerates the healing process in tooth sockets of rats. $\mathrm{Ar}$ Oral Biol 53: 1155-1162.

Montjovent MO, Burri N, Mark S, et al. 2004; Fetal bone cells for tissue engineering. Bone 35: 1323-1333.

Montjovent MO, Mathieu L, Hinz B, et al. 2005; Biocompatibility of bioresobable poly (L-lactic acid) composite scaffold obtained by supercritical gas foaming with human fetal bone cells. Tissue Eng 11: 1640-1649.

Montjovent M-O, Mathieu L, Schmoekel H, et al. 2007; Repair of critical size defects in the rat cranium using ceramic-reinforced PLA scaffolds obtained by supercritical gas foaming. J Biomed Mater Res 83A: 41-51. Montjovent M-O, Silke M, Mathieu L, et al. 2008; Human fetal bone cells associated with ceramic-reinforced PLA scaffolds for tissue engineering. Bone 42: 554-564.

Montjovent MO, Bocelli-Tyndall C, Scaletta C, et al. 2009; In vitro characterization of immune-related properties of human fetal bone cells for potential tissue engineering applications. Tissue Eng 15: 1523-1532.

Nikolidakis D, Meijer GJ, Oortgiesen DAW, et al. 2009; The effect of a low dose of transforming growth factor $\beta 1$ (TGF- $\beta 1$ ) on the early bone-healing around oral implants inserted in trabecular bone. Biomaterials 30: 94-99.

Patino MG, Neiders ME, Andreana S, et al. 2002; Collagen as an implantable material in medicine and dentistry. $J$ Oral Implantol 28: 220-225.

Pfeffer N, Kent J. 2006; Consent to the use of aborted fetuses in stem cell research and therapies. Clin Ethics 1: 216-218.

Pioletti DP, Montjovent MO, Zambelli P-Y, et al. 2006; Bone tissue engineering using foetal cell therapy. Swiss Med Wkly 136: $557-560$

Quintin A, Hirt-Burri N, Scaletta C, et al. 2007; Consistency and safety of fetal cell banks for research and clinical use: preliminary analysis of fetal skin banks. Cell Transpl 16: 675-684.

Ramelet A-A, Hirt-Burri N, Raffoul W, et al. 2009; Chronic wound healing by fetal cell therapy may be explained by differential 
gene profiling observed in fetal versus old skin cells. Exp Gerontol 44: 208-218.

Strong DM, Friedlaender GE, Tomford WW, et al. 1996; Immunologic responses in human recipients of osseous and ostochondral allografts. Clin Orthoped Relat Res 326: 107-114.

Tobita M, Uysal AC, Ogawa R, et al. 2008; Periodontal tissue regeneration with adipose-derived stem cells. Tissue Eng 14: 945-953.
Trommelmans L, Selling J, Dierickx K. 2007; A critical assessment of the directive on tissue engineering of the European Union. Tissue Eng 13: 667-672.

Trommelmans L, Selling J, Dierickx K. 2008; Informing participants in clinical trials with ex vivo human tissue-engineered products: what to tell and how to tell it? J Tissue Eng Reg Med 2: 236-241.

Wang HL. 1998; Guided tissue regeneration. Dent Clin North Am 42: 505-523.
Weinand C, Pomerantseva I, Neville CM, et al. 2006; Hydrogel- $\beta$-TCP scaffolds and stem cells for tissue engineering bone. Bone 38: 555-563.

Yildirim M, Spiekermann H, Biesterfeld S, et al. 2000; Maxillary sinus augmentation using xenogenic bone substitute material Bio-Oss in combination with venous blood. Clin Oral Impl Res 11: 217-229.

Younger EM, Chapman MW. 1989; Morbidity at bone graft donor sites. $J$ Orthop Trauma 3: 192-195. 